\title{
Bandwidth Consumption Control and Service Differentiation for Video Streaming
}

\author{
Can Basaran, Kyoung-Don Kang, Mehmet H. Suzer \\ Department of Computer Science \\ State University of New York at Binghamton \\ \{cbasaran,kang,msuzer\}@cs.binghamton.edu
}

\author{
Ki-Sook Chung, Hae-Ryong Lee, Kwang-Roh Park \\ Home Network Technology Group
}

Electronics and Telecommunications Research Institute

\{kschung,hrlee,krpark\}@etri.re.kr

\begin{abstract}
Multimedia streaming is resource demanding. It may starve other applications such as file transfer sharing the network, for example, in a smart home. To address the problem, we apply fuzzy logic control to bound the bandwidth consumption of multimedia streams. We also differentiate the video quality for streams with different levels of importance. We have implemented our transmission rate control and service differentiation schemes and evaluated them in our department network where a number of different applications may coexist at the same time. Performance evaluation results show that our video streaming system can support the specified bit rate bound and differentiate the service to efficiently utilize the limited bandwidth.
\end{abstract}

\section{INTRODUCTION}

For real-time streaming, a streaming server may take a greedy approach, in which it simply transmits every frame with the highest possible quality. As a result, however, multimedia streams may starve other applications, e.g., file transfer, web surfing, email, and instant messaging in smart homes and buildings. Thus, a system administrator may specify the bit rate bound for streaming. Also, different videos may have different levels of importance. For instance, a live stream of the cradle in the baby's room may have a higher degree of importance than a movie. Hence, the quality of videos need to be differentiated according to their importance to efficiently utilize limited resources.

To bound the bandwidth usage of streaming, in this paper, we leverage the layered encoding technique, in which a video frame consists of a base layer and multiple enhancement layers. We always transmit the base layer, because it is required to display a scene. However, under overload, we degrade the video quality by dropping high enhancement layers without affecting the underlying layers, if necessary, to support the specified bit rate bound. A key challenge is how to determine how many enhancement layers to transmit for concurrent video streams to support the specified bit rate bound. This is not a trivial problem, since the size of a frame consisted of a base layer and enhancement layers may significantly vary in time depending on the complexity of the scenes and their interrelations in a video. Figure 1 is a plot of base-layer size for a single stream and shows the varying nature of a frame size clearly. Also, new multimedia streaming sessions may start, while others may terminate, further increasing the complexity of bit rate control.

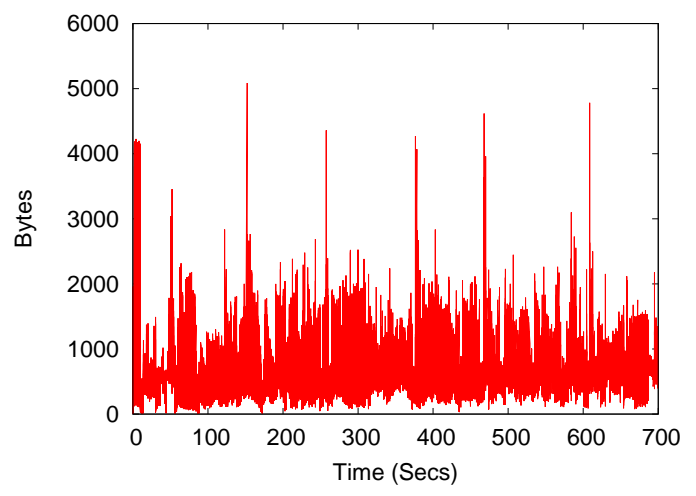

Fig. 1. Size of the base-layer over time

In this paper, we present our QoS-aware video streaming framework (qVSF) implemented on top of an open source video streaming server, QStream [1]. We apply fuzzy logic control to support the specified end-to-end bit rate bound at the node with the bottleneck link that suffers the most significant network congestion, since it may have the lowest physical bandwidth and/or the largest amount of streaming data to deliver. The controller at the bottleneck node informs streaming servers, i.e., stream data sources, how many enhancement layers they can transmit during the next sampling period. If streams going through the bottleneck link have different levels of importance, qVSF differentiates the service by allowing more important stream sources to transmit more enhancement layers. To this end, we apply fuzzy control theory, since it is known to be very effective to support the desired performance when the system model is complex and nonlinear [2]. Fuzzy control theory provides formal techniques to represent, manipulate, and implement human experts' heuristic knowledge for controlling a plant, e.g., a video streaming application, via ifthen rules rather than relying on a mathematical system model developed for specific workloads, e.g., specific video streams.

$\mathrm{qVSF}$ is implemented as a middleware without requiring to change routers or switches. For performance evaluation, we have run experiments across the shared department network in the Department of Computer Science at SUNY Binghamton where a considerable number of different applications usually coexist at the same time. The performance evaluation results show that qVSF can support the specified end-to-end bit rate 


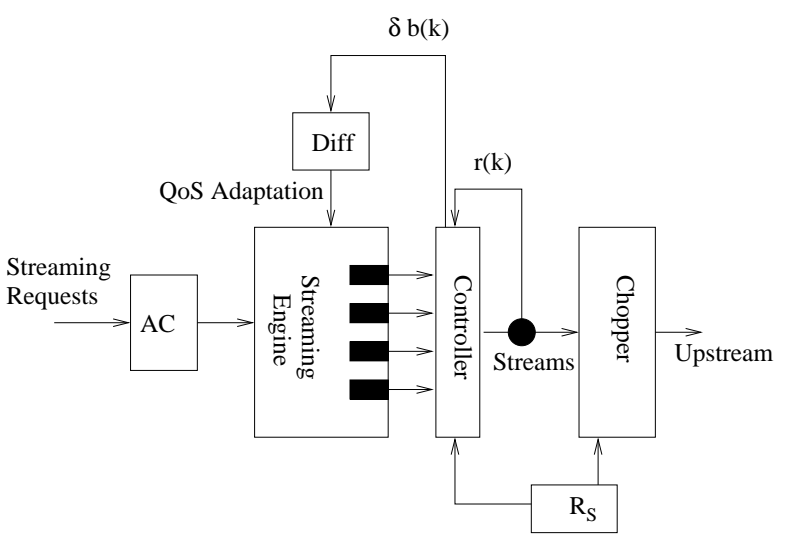

Fig. 2. QoS-Aware Streaming Structure

bound. For efficient utilization of the bounded bandwidth, it also differentiates the service by transmitting more enhancement layers for more important streams without severely degrading the visual quality of low priority video streams.

The rest of the paper is organized as follows. Section II describes the architecture of qVSF as well as the rate control and service differentiation schemes. Section III describes the design of our fuzzy control scheme. Section IV presents the performance evaluation results. Key related work is reviewed in Section V. Finally, Section VI concludes the paper and discusses future work.

\section{System Architecture And Service DIFFERENTIATION}

In this paper, we adopt the UDP protocol widely used for video streaming. In our approach, frames are transmitted in an EDF (earliest deadline first) manner. Thus, the frame with the shortest playtime deadline is transmitted first. Figure 2 shows the structure of qVSF that consists of the admission controller (AC), streaming engine, fuzzy controller, service differentiator (Diff), and chopper. A description of these components and their interactions follows.

Admission Control. A straightforward approach to admission control may rely on the peak bit rate requirement of a video stream. However, this simplistic approach is too pessimistic. As the frame size may significantly vary over time, a reservation approach based on the peak bit rate can waste a large fraction of the reserved bandwidth for most of the time. Also, carefully dropping a subset of enhancement layers under overload might not severely degrade the user perceived QoS, while considerably reducing the bandwidth consumptions. For these reasons, we take an optimistic approach to admission control that accepts a streaming request as long as its average bit rate computed at the video encoding time can be met without affecting the existing streams. Suppose there are $m$ multimedia streams already in the system and stream $j(1 \leq j \leq m)$ requires $r_{j}$ average bps (bits per second). When a new streaming request arrives, the AC in Figure 2 admits it, if $r_{n e w}+\sum_{j=1}^{m} r_{j} \leq R_{s}$ where $r_{\text {new }}$ is the required average bit rate of the newly incoming request and $R_{s}$ is the bit rate bound specified by a system administrator.

Fuzzy Control. The stream engine periodically transmits frames. Even if the sum of the average bit rates does not exceed the specified bit rate bound, several streams may need to transmit large frames at the same time. As a result, the rate bound could be violated. In Figure 2, the fuzzy controller computes the bit rate control error:

$$
e(k)=R_{s}-r(k)
$$

where $r(k)$ is the bandwidth used during the $k^{t h}(k \geq 1)$ sampling period, i.e., [(k-1)P, kP), where $P$ is the sampling interval ( $=1 \mathrm{~s}$ in this paper). Our fuzzy controller also computes the change of the error:

$$
\delta e(k)=e(k)-e(k-1) .
$$

Based on $e(k)$ and $\delta e(k)$, the controller computes the required bandwidth usage adaptation $\delta b(k)$ at the $k^{\text {th }}$ sampling instant, i.e., $k P$. When $r(k)>R_{s}$, the rate control signal $\delta b(k)$ is negative to reduce the bandwidth usage in the $(k+1)^{t h}$ sampling period and vice versa. A detailed discussion of the fuzzy control signal computation is given in Section III.

Chopping. Popular video encoding techniques such as MPEG or SPEG result in variable sizes of frames in a video. Thus, there could be times at which large bursts of multiple streams overlap. As a result, a feedback controller may not be able to completely avoid overshoots-bandwidth consumptions exceeding the specified bound. One can fine-tune the controller for specific workloads to minimize potential overshoots. However, this approach is against the qVSF design concept, since fine-tuned controllers may perform poorly for different workloads. To address this problem, qVSF relies on fuzzy control theory, which does not require a precise mathematical model of the controlled system or fine-tuning. Also, qVSF has a chopper to constantly monitor the transmission rate and drop enhancement layers when there is an overshoot due to potentially insufficient QoS adaptation directed by the fuzzy controller. For these reasons, in Figure 2, we intentionally place the chopper outside the fuzzy control loop to have no overshoot in qVSF. Also, to enhance the perceived video quality via effective QoS adaptation, the fuzzy controller tries to reduce the error measured before potential chopping.

Service Differentiation. The importance of a stream can be determined based on many factors such as the number of individuals interested in the stream, the importance of the content itself, or monetary costs for streaming paid by subscribers. In this paper, we assume that weights, i.e., importance levels, of streams are given by users based on their subjective notion of relative importance among video streams. Given the bandwidth usage control signal $\delta b(k)$, we adapt QoS based on the relative weights at times of stream bursts to support the bit rate bound, while differentiating services for efficient bandwidth utilization.

In the current version of qVSF, each client specifies an initial weight when it requests a multimedia stream. For stream $j$, let $w_{j}^{i}$ and $w_{j}^{e}$ represent the initial weight and effective 
weight, respectively. The effective weight is computed: $w_{j}^{e}=$ $w_{j}^{i} / \sum_{j=1}^{n} w_{j}^{i}$ where $n$ is the total number of streams. When a new streaming request is accepted, the effective weights are recomputed. Thus, it always holds that $\sum_{j=1}^{n} w_{j}^{e}=1$.

Effective weights are used to distribute the control signal $\delta b(k)$ to streams. When the network is congested, low weight streams are forced to drop more enhancement layers. When the network is underutilized, higher importance streams can transmit more enhancement layers. Separately, the priority mapper of QStream [1] assigns a priority to each layer ranging from 0 to 15 where 15 is the highest priority. Note that 16 priority levels are specific to QStream. Our approach is not limited to QStream, but it is generally applicable to bandwidth control and service differentiation for real-time streaming.

Priorities are assigned according to the utility of a layer estimated in terms of the user perceived QoS considering the type of the frame (i.e., I, B, or P) and its rank in the layer hierarchy. To support bandwidth control and service differentiation at the same time, qVSF performs the following procedure at the $k^{t h}(\geq 1)$ sampling instant.

1) Compute the bandwidth control signal $\delta b(k)$.

2) Let $N_{p}$ represent the number of priority levels. In QStream, $N_{p}=16$. Compute the transmission threshold for $s_{j}$, i.e., stream $j: \theta_{j}(k)=\left\lceil\theta_{j}(k-1)-\delta b(k)\right.$. $\left.f\left(\delta b(k), w_{j}^{e}\right) \cdot\left(N_{p}-1\right)\right\rceil$ where

- $f\left(\delta b(k), w_{j}^{e}\right)=w_{j}^{e}$ if $\delta b(k) \geq 0$.

- $f\left(\delta b(k), w_{j}^{e}\right)=1-w_{j}^{e}$ otherwise.

If $\theta_{j}>N_{p}-1$, set $\theta_{j}=N_{p}-1$. If $\theta_{j}<0$, set $\theta_{j}=0$. Note that $\forall j \theta_{j}(0)=N_{p}-1$.

3) During the $(k+1)^{t h}$ sampling period, transmit an enhancement layer of $s_{j}$, if its QStream priority is higher than or equal to $\theta_{j}(k)$. Otherwise, drop it. For example, if $\theta_{j}(k)=14$, qVSF transmits only the base layer, which always has the highest priority $N_{p}-1$ and the enhancement layer with priority $N_{p}-2(=14)$ for $s_{j}$ 's frame(s) during the $(k+1)^{t h}$ sampling period. Since $\forall j, k \theta_{j}(k) \leq N_{p}-1$ in step 2, the base layer is always transmitted. Also, the higher $w_{j}$, the lower $\theta_{j}(k)$ in step 2. Thus, qVSF transmits more (or drops fewer) enhancement layers for a higher weight stream when the network is underutilized (or overloaded). Further, $\theta_{j}(k) \geq \theta_{j}(k-1)$ (or $\theta_{j}(k) \leq \theta_{j}(k-1)$ ) when $\delta b(k-1)$ and $\delta b(k)$ are both negative (or positive) due to continuous overload (or underutilization) conditions. Hence, fewer enhancement layers are transmitted when the network is continuously overloaded and vice versa.

Multi-hop Settings. Figure 3 shows a possible multi-hop layout of qVSF. In this figure, the intermediate relay node 2 has the most demanding resource requirements to forward real-time streams originating at the servers $1,2, \ldots, \mathrm{N}+1$. As a result, the network link between the relay node 2 and its downstream client becomes the bottleneck. In this case, the controller at the relay node 2 takes control of the bandwidth usage control, while the other nodes turn off their controllers and follow the control decisions made by the bottleneck node.

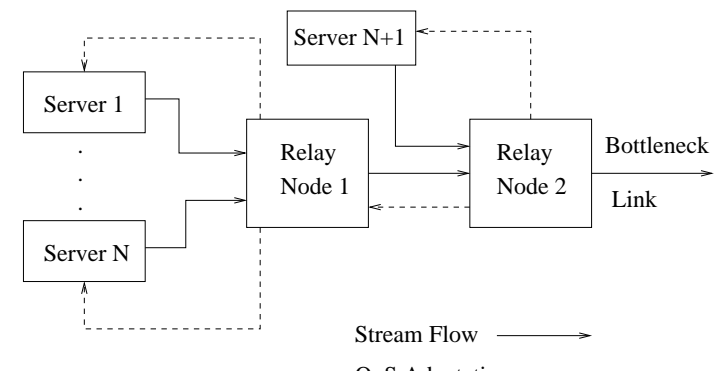

QoS Adaptation

Fig. 3. Multi-Hop qVSF System

On the other hand, each node always runs its own chopper to ensure that the bit rate on its outgoing link does not exceed $R_{s}$. Thus, the end-to-end bandwidth usage does not exceed $R_{s}$. In summary, via fuzzy control and chopping, we ensure that the UDP traffic rate for streaming conforms to the bit rate bound specified by the system administrator. By supporting rate control and service differentiation, our work could provide a basis for important real-time and non-realtime applications such as network services in smart homes and buildings. A system administrator can allocate the bandwidth with the confidence that the allocated bandwidth limits and service differentiation policy will be enforced by our approach. A thorough investigation of bandwidth allocation between different types of applications, e.g., video streaming and file transfer, in smart environments is reserved for future work.

\section{Fuzzy Control of the Transmission Rate}

In this section, the design of our fuzzy logic controller is discussed. For the clarify of presentation, we convert the rate control problem to the problem of controlling the utilization of the bottleneck link. To achieve the target utilization, our fuzzy controller computes the workload control signal based on the error $e(k)$ and change of the error $\delta e(k)$ redefined as follows:

$$
\begin{gathered}
e(k)=U_{s}-u(k)=U_{s}-r(k) / R_{s} \\
\delta e(k)=e(k)-e(k-1)
\end{gathered}
$$

where the utilization $u(k)$ is the total bandwidth utilization in the $k^{t h}$ sampling period. In this paper, we set the target utilization $U_{s}=1$ to fully utilize $R_{s}$. As discussed before, there is no overshoot due to possible chopping.

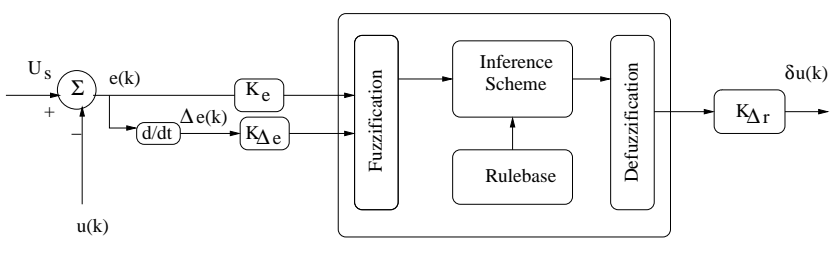

Fig. 4. Fuzzy Controller

Based on $e(k)$ and $\delta e(k)$ computed in Eq. 3 and Eq. 4, the fuzzy rate controller computes the required utilization adjustment $\delta u(k)$ for the next sampling period. $K_{e}, K_{\delta e}$, and 
$K_{\Delta r}$ in Figure 4 are control gains tuned by trial and error. Specifically, we choose $K_{e}=1.0, K_{\delta e}=0.5$, and $K_{\Delta r}=$ 0.1 . Intuitively, $e(k)$ at the $k^{\text {th }}$ sampling instant needs to be fully considered, while $\delta e(k)$ and (especially) $\delta u(k)$ need to be damped to avoid oscillations. Note that key to effective fuzzy control is good understandings and insights of the controlled system behaviors and corresponding rule-base design [2], [3]. Flexible tuning is a virtue of fuzzy control.

\section{A. Fuzzy Utilization Control}

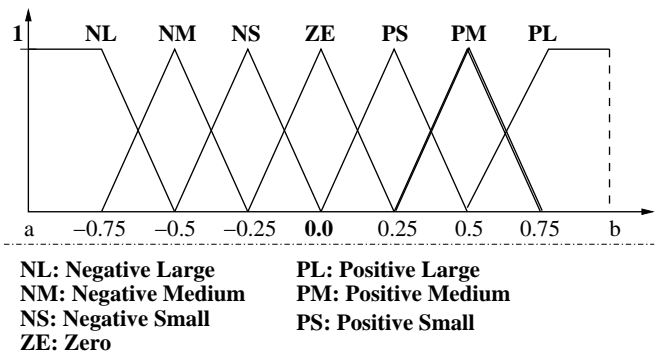

Fig. 5. Membership Functions

The universe of discourse (UD) is the domain of the inputs to a fuzzy controller. In Figure 5, the UD is $[a, b]$. Note that $a \rightarrow-\infty$ for both $e(k)$ and $\delta e(k)$. If there is no bandwidth usage control, a streaming engine may consume the bandwidth with no bound. As a result, the utilization error (Eq 3) could theoretically go to the negative infinity. In practice, it will consume the maximum bandwidth physically available in the worst case and, therefore, $a$ will be bounded. By having $a \rightarrow$ $-\infty$, however, our UD becomes independent of the physical bandwidth limit, which may change from network to network.

Note that $b=1$ for $e(k)$, since the maximum utilization set-point $U_{s}=1$ and $U_{s} \geq u(k)$ when $e(k) \geq 0$ in Eq 3. In contrast, $b \rightarrow \infty$ for $\delta e(k)$, because, for example, $e(k)=0$ and $e(k-1) \rightarrow-\infty$ due to theoretically unbounded bandwidth consumption. Finally, the UD $=[-1,1]$ for the utilization control signal after defuzzification in Figure 4 due to Eq 5 to be discussed shortly. Thus, the UD of $\delta u(k)$ is $\left[-K_{\Delta r}, K_{\Delta r}\right]$, i.e., [-0.1, 0.1] in this paper. By limiting the amplitude of the control signal to a small range, we can reduce potential performance oscillations due to too large (positive or negative) control signals. In summary, the UDs for $e(k), \delta e(k)$, and $\delta u(k)$ are $(-\infty, 1],(-\infty, \infty)$, and $[-0.1,0.1]$ in this paper.

Linguistic variables describe the inputs and output(s) of a fuzzy controller. The fuzzification interface in Figure 4 converts $e(k)$ and $\delta e(k)$ to the corresponding linguistic values defined next.

A linguistic variable such as the error (fuzzified $e(k)$ ) or change in error (fuzzified $\delta e(k)$ ) is associated with linguistic values to describe characteristics of the variables. Figure 5 shows linguistic values for the linguistic variables error, change in error, and workload control signal used in this paper.

A linguistic rule is a "IF premise THEN consequent" rule used to map the inputs to output(s) of a fuzzy controller.
For example, if error $=N L$ (negative large) and change in error $=N L$ at the $k^{t h}$ sampling instance, then the link is overloaded, i.e., $U_{s}<u(k)$, and the degree of overload is increasing significantly. Thus, the corresponding rule generates a $N L$ signal to reduce the workload by a large amount.

The rule-base in Figure 4 has a set of IF-THEN rules dictating how to achieve $U_{s}$ according to the error and change in error. The inference mechanism in Figure 4 evaluates which control rules are relevant at the current time to compute the fuzzy control signal by looking up the rule-base table based on the fuzzified $e(k)$ and $\delta e(k)$ values.

The defuzzification interface in Figure 4 converts the fuzzy control signal reached by the inference mechanism to the control signal $\delta u(k)$ expressed as a real number. $\delta u(k)$ is the input to the plant, i.e., the controlled real-time streaming system, which is required to adjust the bit rate consumption according to $\delta u(k)$ by QoS adaptation.

The horizontal axis of Figure 5 represents $e(k)$ or $\delta e(k)$ and the vertical axis indicates the membership value. A membership function (MF) quantifies the certainty an $e(k)$ (or $\delta e(k)$ ) value to be associated with a certain linguistic value. For MFs, we use symmetric triangles of an equal base and $50 \%$ overlap with adjacent MFs. A triangular MF is one of the most popular MF's in fuzzy control [2]. For example, suppose $e(k)=0.25$. In Figure 5, $\mu_{P S}(0.25)=1$; that is, 0.25 belongs to the fuzzy set PS with certainty 1 .

Based on the fuzzified $e(k)$ and $\delta e(k)$, the inference mechanism in Figure 4 determines which rules to apply at the $k^{t h}$ sampling instance. For example, suppose $e(k)$ $=0.25$ and $\delta e(k)=0.0625$. According to Figure 5, the certainty $\mu_{P S}(0.25)=1$ for $e(k)$ and $\mu_{Z E}(0.0625)=0.75$ and $\mu_{P S}(0.0625)=0.25$ for $\delta e(k)$. To compute the certainty value of the premise in the corresponding IF premise THEN consequent rule(s), we take the minimum between the certainty values of $e(k)$ and $\delta e(k)$, following the one of the most common approaches [2], [3], [4]. Thus, $\mu$ (error, change in error $)=\mu(P S, Z E)=\min \{1,0.75\}=0.75$ and $\mu(P S, P S)=$ $\min \{1,0.25\}=0.25$.

By referring to our rule base in Table I, the inference engine finds that rule $(P S, Z E)=P S$ and $\operatorname{rule}(P S, P S)=P M$. Let $\mu(i, j)$ denote the membership function and $c(i, j)$ denote the center of the MF of the consequent of the rule $(i, j)$. For triangle MFs, the center is the value on the $\mathrm{x}$ axis at the middle of the triangle [2]. For example, in Figure 5, the center of $P S$ and $P M$ is 0.25 and 0.5 , respectively. Then the fuzzy utilization control output is:

$$
\delta u(k)=\frac{\sum_{i, j} c(i, j) \cdot \mu(i, j)}{\sum_{i, j} \mu(i, j)}
$$

In the previous example, the defuzzified control signal $\delta u(k)=\frac{0.25 \cdot 0.75+0.5 \cdot 0.25}{0.75+0.25}=0.3125$. For more information about fuzzy control, readers are referred to [2]. A discussion of key ideas behind our rule base design follows. 


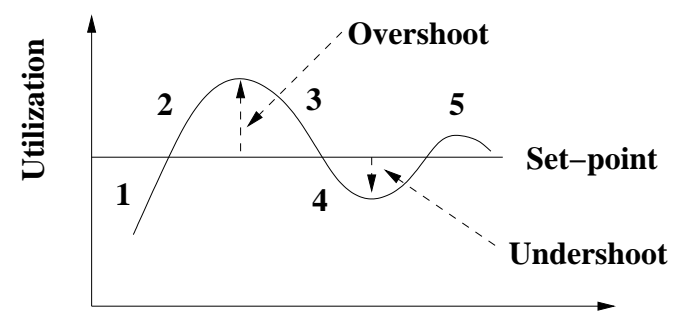

Time

Fig. 6. Utilization Control Characteristics

\begin{tabular}{|l|l|l|l|l|l|l|l|}
\hline e/ $\delta e$ & NL & NM & NS & ZE & PS & PM & PL \\
\hline NL & NL & NL & NL & NL & NM & NS & ZE \\
\hline NM & NL & NL & NL & NM & NS & ZE & PS \\
\hline NS & NL & NL & NM & NS & ZE & PS & PM \\
\hline ZE & NL & NM & NS & ZE & PS & PM & PL \\
\hline PS & NM & NS & ZE & PS & PM & PL & PL \\
\hline PM & NS & ZE & PS & PM & PL & PL & PL \\
\hline PL & ZE & PS & PM & PL & PL & PL & PL \\
\hline
\end{tabular}

TABLE I

UTILIZATION CONTROL RULES

\section{B. Fuzzy Rule Base}

As shown in Figure 6, there are five zones that characterize the utilization controller's action, from which we derive the rule-base for fuzzy utilization control described in Table I:

1) If $e(k)$ is positive and $\delta e(k)$ is negative, the actual utilization is smaller than the target utilization, but it comes closer to the set-point. The controller applies a small signal to avoid a potential overshoot while increasing the utilization.

2) If $e(k)$ is negative and $\delta e(k)$ is negative, the actual utilization is larger than the set-point and it is further increasing. The controller must reduce the load to reverse the current trend.

3) $e(k)$ is negative and $\delta e(k)$ is positive, the actual utilization is higher than the target utilization, but it comes closer to the set-point. Since the actual utilization is converging to the target, the controller applies a small signal to avoid a potential undershoot, i.e., underutilization, while reducing the utilization.

4) $e(k)$ is positive and $\delta e(k)$ is positive, the actual utilization is lower than the set-point and it is further decreasing. Thus, the controller increases the utilization to reverse the current trend.

5) If $|e(k)|<\epsilon$ and $|\delta e(k)|<\epsilon$ where $\epsilon$ is a predefined small positive real number, the utilization converges to the set-point and $|e(k)|$ and $|\delta e(k)|$ are small. Hence, the utilization adjustment $\delta u(k)=0$.

Overall, our fuzzy logic control is lightweight, only requiring a small rule-base, efficient table look-ups, and control signal computation (Eq $3-\mathrm{Eq} 5)$.

\section{PERFormance EVAluation}

The results given in this section are taken from a streaming session during which four clients are served. The duration of the session was 13 minutes. Client requests were sent at arbitrary times within the first minute of the session. Each client requested a different video from the server over our department network. The set-point for transmission rate is set as $2 \mathrm{Mbps}$, while the four streamed videos require approximately $1.8 \mathrm{Mbps}$ in average. The choice of the set-point was an arbitrary one in our case, driven by our desire to create a harsh environment. For the same settings, QStream [1] without our QoS management schemes often consumes more than $4 \mathrm{Mbps}$. We have considered different settings too. However, due to space limitations, we only present key performance evaluation results.

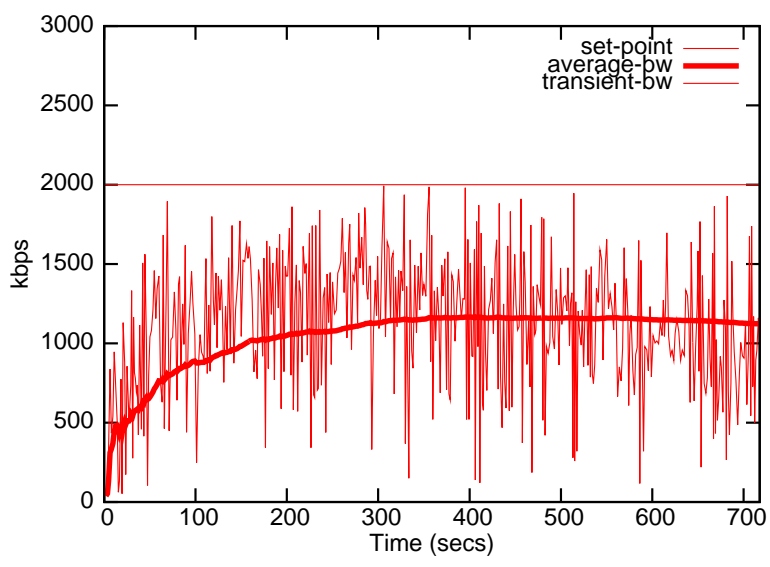

Fig. 7. Transient and average bandwidth consumption (4 streams)

Figure 7 shows the bandwidth usage of the streaming server for the entire session. Transient bandwidth is the transmission rate of the server measured every second. The average bandwidth usage at time $t$ is the average bandwidth consumption over the time period $[0, t]$. As shown in the figure, cooperation between the controller and chopper eliminates overshoots for the entire streaming session period. Without chopping, our fuzzy controller experiences approximately 10 overshoots in the 13 minute experiment and the largest transient bandwidth usage was approximately $2.5 \mathrm{Mbps}$. Moreover, due to the nature of video streaming, undershoots may happen when the frames of multiple streams to be be transmitted are small. For example, the current frames could be relatively small when they are similar to their previous frames. Since the peak bursts are kept at the set point and the bandwidth consumption is smaller than the set-point except the peaks, the average transmission rate is lower than the specified bound in qVSF. In the future, we will investigate an approach to further increase the efficiency of bandwidth utilization without violating the specified bit rate bound.

Figure 8 shows the average number of enhancement layers transmitted for four individual streams. According to this figure, our QoS management scheme effectively differentiates services. For the stream with smallest effective weight of 0.1 , 


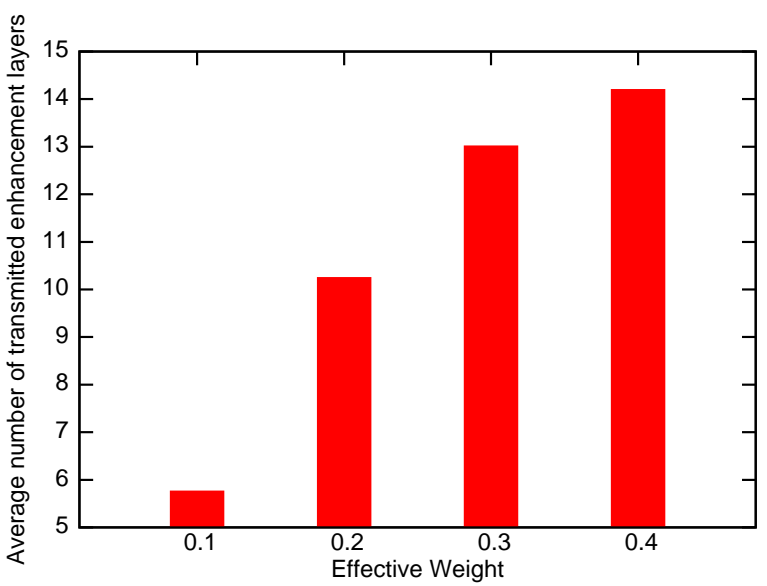

Fig. 8. Average number of transmitted enhancement layers per stream

6 enhancement layers were sent in average. For the stream with the highest effective weight of 0.4 , an average of 14 enhancement layers out of 15 enhancement layers were sent. Note that QoS for each stream is not perfectly proportional to its effective weight. There are two reasons for this. First, a base-layer is larger than its enhancement layers and layer sizes also vary from frame to frame. Since our service differentiation policy is based on bandwidth consumptions, we expect total bytes sent per stream to be proportional to its effective weight rather than the number of transmitted layers. Second, a control signal cannot be applied to saturated streams; that is, when a stream is already at the highest/lowest acceptable QoS level, a positive/negative control signal will not apply to that particular stream. A thorough investigation of a more sophisticated service differentiation scheme is reserved for future work. Overall, we have observed that our approach can support visually acceptable quality for even the lowest priority stream by always streaming the base frame [5].

\section{RELATED WORK}

Our bit rate management and service differentiation schemes are implemented atop QStream [6]. QStream supports realtime video streaming by EDF (earliest deadline first) scheduling of frames based on their playtime deadlines and synchronization between the client and server. qVSF significantly extends QStream to support the specified bandwidth limit and service differentiation.

A fuzzy controller [7] determines the emitted QoS level of video streams. The error value fed into the fuzzy controller is the difference between the stream quality emitted from the server and the stream quality perceived at the client. They aim to find the point where the difference between the emitted QoS level and the perceived one is zero. However, bit rate control and service differentiation are not considered in their work.

A fuzzy logic based cross-layer video streaming solution for wireless media is presented in [8]. The feedback is received from the underlying network stack to change the bit rate of a video according to link conditions. However, their work only considers a single stream. Also, it only considers to perform fuzzy logic control and QoS adaptation at the source node that encodes the video.

Stok et al. [9] propose a system that can react to network condition variations in a wireless home network. They use TCP for streaming and apply priority based rules to delete lower priority packets from the buffer if the buffer overflows. Resource allocation is a complementary problem to our work presented in this paper and it is reserved for our future work.

\section{CONCLUSIONS AND Future Work}

If greedy QoS management techniques are applied, realtime video streams may starve other applications such as file transfer. Also, different video streams may have different importance levels to users. To address these research issues, we present a real-time video streaming system called qVSF in this paper. We present optimistic admission control and fuzzy logic control schemes developed to support the specified bandwidth usage bound. We present a service differentiation scheme to efficiently utilize the limited bandwidth for streamed videos with different priorities. Moreover, we have implemented qVSF and evaluated it in a local area network shared with different users and applications. qVSF supports the specified transmission rate bound and effectively differentiates the service without unduly degrading the quality of a low priority video.In the future, we will investigate more advanced approaches for real-time video streaming.

\section{ACKNOWLEDGMENT}

This work was supported, in part, by the IT R\&D program of MIC/IITA [2007-S010-01, A development of Ubiquitous Home Media Service System based on SMMD] and a NSF grant CNS-0614771.

\section{REFERENCES}

[1] “QStream Home," http://qstream.org.

[2] K. M. Passino and S. Yurkovich, Fuzzy Control. Addison-Wesley, 1998.

[3] R. K. Mudi and N. R. Pal, "A Self-Tuning Fuzzy PI Controller," Fuzzy Sets and Systems, vol. 115, no. 2, pp. 327-338, October 2000.

[4] J. Wei and C.-Z. Xu, "eQoS: Provisioning of Client-Perceived End-toEnd QoS Guarantees in Web Servers," IEEE Transactions on Computers, vol. 55, no. 12, pp. 1543-1556, December 2006.

[5] C. Basaran, K.-D. Kang, M. H. Suzer, K. Chung, H.-R. Lee, and K.R. Park, "Bandwidth Consumption Control and Service Differentiation for Video Streaming," Department of Computer Science, SUNY Binghamton, Tech. Rep., 2008, CS-TR-08-KD01 (www.cs.binghamton.edu/ kang/can08tr.pdf).

[6] C. Krasic, J. Walpole, and W.-C. Feng, "Quality-Adaptive Media Streaming by Priority Drop," in NOSSDAV '03, 2003.

[7] C. Koliver, K. Nahrstedt, J.-M. Farines, J. da Silva Fraga, and S. A. Sandri, "Specification, Mapping and Control for QoS Adaptation," RealTime Systems, vol. 23, no. 1-2, pp. 143-174, 2002.

[8] J. Vehkaperä, M. Myllyniemi, and J. Peltola, "Fuzzy Logic-Based CrossLayer Controller for Scalable Video Streaming," in IMSA'07, 2007.

[9] P. van der Stok, D. Jarnikov, S. Kozlov, M. van Hartskamp, and J. J. Lukkien, "Hierarchical Resource Allocation for Robust In-Home Video Streaming," Journal of Systems and Software, vol. 80, no. 7, pp. 951-961, 2007. 